patients, but also the burden of healthcare costs.

\title{
OC37 - Adolescents with cystic fibrosis: their perspective
}

\author{
Maria Conceição Reisinho (Portugal)ª́r Bárbara Gomes (Portugal) \\ 1 - Escola Superior de Enfermagem do Porto
}

Theme: Complex health care and chronic disease management.

Keywords: Adolescent, cystic fibrosis, transitions.

Introduction: The adolescent with cystic fibrosis suffers from organic distresses and requires support to deal with the physical symptoms and the psychosocial adaptation.

Aim: Realize the experience of growing up with cystic fibrosis from the adolescent point of view.

Methodology: Data grounded theory as investigation method and interpretation by Meleis Theory. Study group with 16 adolescents and data collected by interview.

Results: From the content analysis of the interviews, we established that the adolescents were living development and health-illness type transitions. We identified the dimension 'developing trust and coping' as adjustment strategy to new contexts.

Discussion: The adolescents' speeches reflected behaviors of involvement in the transition process, being a positive result to the psychosocial development and positive life experience at this stage.

Conclusion: The follow up from healthcare professionals may transform a stressful situation into a state of trust. It can also reveal and pinpoint resource strategies to enable adaptation to new circumstances.

\section{OC38 - Beyond boundaries: inter-professional simulation in children and young people's nursing - three pilot projects}

Dave Clarke (United Kingdom) ${ }^{1}$

${ }^{1}$ Cardiff University

Theme: Nursing education, management and leadership.

Keywords: Inter-professional, medical students, occupational therapy students, simulation. Inter-professional collaboration is essential for safe, high-quality, patient-centered care (Shanahan and Lewis 2015). In order to set the stage for inter-professional collaboration, inter-professional education should be incorporated into the learning experience of health professionals (Reese et al 2010).

In this presentation I will share the development of two undergraduate inter-professional education pilot projects and the evaluations from these. This comprised of two projects: 
children's nursing students and occupational therapy students, and children's nursing students and fourth-year medical students. Each had a different focus: the first is based upon assessment of a child in a community simulation, whilst the second focused on the inter-professional assessment of the acutely ill child.

I will present the results of these two successful inter-professional simulation innovations and then discuss the future of inter-professional education. I will conclude by presenting our current project which is a practice-based inter-professional education intervention involving up to eight professional groups.

\title{
OC39 - The efficacy of a participatory approach in reducing pain related to venepuncture in children
}

\author{
Andrea Gazzelloni (Italy)'; Mariagrazia Maio (Italy)2 ; \\ Maria Romano (Italy) ${ }^{2}$; Iolanda Marcone (Italy) ${ }^{2}$; Franca Marino \\ $(\text { Italy })^{2}$; Monia Cosima Labalestra (Italy) ${ }^{2}$
}

${ }^{1}$ Bambino Gesù Children's Hospital; ${ }^{2}$ 'San Carlo' Hospital - Potenza

Theme: Ethical issues: dignity and humanity.

Keywords: Children, ice, illustration, pain, venepuncture.

Introduction: Venepuncture represents traumatic experience in childhood due to pain and discomfort.

Aims: To compare the efficacy of a participatory approach with preliminary ice application to the skin.

Methods: Two age-groups (respectively 3-7 and $\geq 8$ years) were considered during dayhospital, hospitalization, and ER accesses. Venepuncture was described to patients with fables or illustration according to the age-group. Ice or cool-water-pack was randomly applied to skin for one minute before venepuncture. Pain measurement scales were Wong-Baker and VAS.

Results: Preliminary data (55 patients) showed that the 3-7 years age group had better pain tolerance with a participatory approach, whilst ice was better in the older group. Generally previous venepunctures seem to worsen pain feeling.

Discussion: Imagination seems to influence pain feeling in the 3-7 age group, while ice is more important in the older group. Previous experiences mark negatively successive venepunctures.

Conclusions: Participatory approaches can be cost-effective and influences positively venepuncture in the future. 